\title{
FUZZY CONTROL OF THE PERMANENT MAGNET SYNCHRONOUS MACHINE SINGULARLY PERTURBED FED BY A THREE LEVEL INVERTER
}

\author{
Ahmed Massoum * Abdelkader Meroufel ${ }^{*}$ \\ Patrice Wira ${ }^{* *}$ — Mohammed Karim Fellah ${ }^{*}$
}

\begin{abstract}
In this paper, we present the control technique based on the singular perturbation technique controlled by a fuzzy regulator applied to the permanent magnet synchronous machine (PMSM). This technique applied to the PMSM conducts to a separation of the variables into disjoined subset or two separated models: one having a slow dynamics, and the other a fast dynamics. To ensure certain robustness to the decoupled control system based on these techniques, the control speed and the $I_{d}$ current is carried out by fuzzy regulators. A qualitative analysis of the principal variables evolution describing the behavior of the global system (PMSM-Inverter with MLI-Control) and its robustness is developed by several tests of digital simulation in last stage.
\end{abstract}

K e y w o r d s: PMSM, singular perturbations, fuzzy control, three levels inverter

\section{INTRODUCTION}

The vector control technique permits to compare the PMSM to the separate excitation DC machine. The vector flux must be concentrated on the $D$ axis with $I_{d}$ current null. However the exact knowledge of the rotor flux position poses a precision problem [1]. The technique based on the singular perturbations control technique which makes abstraction with the flux orientation permits to solve this problem.

It also allows, to completely decouple the system in two linear and mono variable subsystems. Thus, it is possible to control independently the speed and the forward current $I_{d}$. The traditional control algorithms (PI or PID) prove to be insufficient where the requirements in performances are very severe. Several methods of control are proposed in the technical literature, among them, the fuzzy control which held our attention by the simplicity of its adjustment algorithm and which is the objective of our work $[2,3]$. The work is composed by a PMSM modelling in the Park frame and an overview of singular perturbation control technique in order to decouple the machine model. Then, a brief outline on the fuzzy control and its application to the speed and the $I_{d}$ current control of the PMSM supplied with an MLI inverter. In the last step, a comment on the results obtained in simulation and a conclusion where we emphasize the interest and the contribution of this method of control.

\section{THE PMSM NONLINEAR MODEL}

With the simplifying assumptions relating to the PMSM, the model of the machine expressed in the reference frame of Park, in the form of state is written as
$[2,3]$

$$
\begin{aligned}
& \dot{x}=F(x)+\sum_{i=1}^{m} g_{i}(x) U_{i} \\
& x=\left(\begin{array}{c}
x_{1} \\
x_{2} \\
x_{3}
\end{array}\right)=\left(\begin{array}{c}
i_{d} \\
i_{q} \\
\text { Omega }
\end{array}\right) ; U_{i}=\left(\begin{array}{c}
u_{1} \\
u_{2}
\end{array}\right)=\left(\begin{array}{c}
v_{d s} \\
v_{q s}
\end{array}\right) \\
& g_{1}=\left(\begin{array}{lll}
\frac{1}{L_{d}} & 0 & 0
\end{array}\right)^{\top} ; g_{2}=\left(\begin{array}{lll}
0 & \frac{1}{L_{q}} & 0
\end{array}\right)^{\top} \\
& F(x)=\left(\begin{array}{l}
f_{1}(x) \\
f_{2}(x) \\
f_{3}(x)
\end{array}\right)= \\
& \left(\begin{array}{c}
\frac{-R}{L_{d}} x_{1}+\frac{p L_{q}}{L_{d}} x_{2} x_{3} \\
\frac{-R}{L_{q}} x_{2}-\frac{p L_{d}}{L_{q}} x_{1} x_{3}-\frac{p \Phi_{f}}{L_{q}} x_{3} \\
\frac{-f}{J} x_{3}+\frac{p\left(L_{d}-L_{q}\right.}{J} x_{1} x_{2}+\frac{p \Phi_{f}}{J} x_{2}-\frac{C_{r}}{J}
\end{array}\right)
\end{aligned}
$$

The variables to be controlled are the current $I_{d}$ and the mechanical speed $\Omega$

$$
y(x)=\left(\begin{array}{c}
y_{1}(x) \\
y_{2}(x)
\end{array}\right)=\left(\begin{array}{c}
h_{(x)} \\
h_{2}(x)
\end{array}\right)=\left(\begin{array}{l}
x_{1} \\
x_{2}
\end{array}\right)=\left(\begin{array}{c}
i_{d} \\
\Omega
\end{array}\right) .
$$

\section{SINGULAR PERTURBATION APPLIED TO PMSM}

This technique conducts to a separation of the original system into disjoined subset or two separated models: one having a slow dynamics, and the other a fast dynamics [7-9].

\footnotetext{
* Department of Electrical Engineering, Faculty of Engineering Science, Djillali Liabes University, 22000 Sidi Bel-Abbes, Algeria, ${ }^{* *}$ Faculty of Sciences and Technics, Haute Alsace University, ahmassoum@yahoo.fr, ameroufel@yahoo.fr, mkfellah@yahoo.fr, patrice.wira@uha.fr
} 

$[7-9]$

The standard form for singular perturbed systems is

$$
\begin{aligned}
\dot{x}_{s} & =f_{1}\left(x_{s}, x_{f}, u, t, \varepsilon\right), \\
\varepsilon \dot{x}_{f} & =f_{2}\left(x_{s}, x_{f}, u, t, \varepsilon\right), \\
y & =h\left(x_{s}, x_{f}\right) .
\end{aligned}
$$

Considering

$$
\begin{aligned}
& x=\left(x_{1}, x_{2}\right)^{\top}=\left(x_{1 s}+x_{1 f}, x_{2 s}+x_{2 f}\right)^{\top}, \\
& u=\left(\begin{array}{ll}
v_{d s} & v_{q s}
\end{array}\right)^{\top}=\left(\begin{array}{ll}
v_{d s s}+v_{d s f} & v_{q s s}+v_{q s f}
\end{array}\right)^{\top}
\end{aligned}
$$

and eliminating the negligible parts of the two sub vectors of the state vector, we will have

$$
\begin{gathered}
x=\left[x_{s}, x_{f}\right] \\
x_{1}=x_{1 s}=x_{s} ; x_{1 f}=0, \\
x_{2}=x_{2 f}=x_{f} ; x_{2 s}=0 .
\end{gathered}
$$

The existence of the small and big time constants constitute an important hint to choose $\varepsilon$, which can be defined as the quotient between these two parameters.

$$
\varepsilon=\frac{\tau_{f}}{\tau_{s}} .
$$

The slow model is calculated by supposing that $\varepsilon=0$

$$
f_{2}\left(x_{s}, x_{f}, u, t, 0\right)=0 .
$$

For the fast model, $\varepsilon$ is set to be zero:

$$
\varepsilon \dot{x}_{f}=f_{2}\left(x_{s}, x_{f}, u, t, \varepsilon\right) .
$$

By considering that the variables $i_{d s}$ and $i_{q s}$ are fast and that $\omega_{r}$ is slow and choosing [7-9]

$$
i_{d s}=i_{d s r}, \quad i_{q s}=i_{q s r}, \quad \omega_{r}=\omega_{r l} .
$$

Defining the state vectors as

$$
\begin{aligned}
& y=\left[\begin{array}{ll}
i_{d s} & \omega_{r}
\end{array}\right]^{\top} ; \quad u=\left[\begin{array}{ll}
v_{d s} & v_{q s}
\end{array}\right]^{\top} ; \\
& x=\left[\begin{array}{lll}
i_{d s} & i_{q s} & \omega_{r}
\end{array}\right]^{\top}
\end{aligned}
$$

We will have

$$
\begin{aligned}
\frac{\mathrm{d} \omega_{r s}}{\mathrm{~d} t} & =\frac{p^{2}}{J} \varphi_{f} i_{q s f}-\frac{p}{J} C_{r}-\frac{f}{J} \omega_{r s} \\
\frac{\mathrm{d} i_{d s f}}{\mathrm{~d} t} & =-\varepsilon \frac{R_{s}}{L_{d}} i_{d s f}+\varepsilon \frac{L_{q}}{L_{d}} \omega_{r l} i_{q s f}+\frac{\varepsilon}{L_{d}} v_{d s}, \\
\varepsilon \frac{\mathrm{d} i_{q s f}}{\mathrm{~d} t} & =-\varepsilon \frac{R_{s}}{L_{q}} i_{q s f}-\varepsilon \frac{L_{d}}{L_{q}} \omega_{r s} i_{d s f}+\frac{\varepsilon}{L_{q}} v_{q s}-\varepsilon \frac{\varphi_{f}}{L_{q}} \omega_{r s} .
\end{aligned}
$$

By rearranging the equations, we obtain:

Slow model

$$
\begin{aligned}
\frac{\mathrm{d} \omega_{r s}}{\mathrm{~d} t}= & \frac{p^{2} \varphi_{f}}{J\left(\frac{R_{s}^{2}}{L_{q} L_{d}}+\omega_{r s}^{2}\right)}\left(\frac{R_{s}}{L_{d} L_{q}} v_{q s}-\frac{R_{s} \varphi_{f}}{L_{d} L_{q}} \omega_{r s}\right. \\
& \left.\quad-\frac{1}{L_{q}} \omega_{r s} v_{d s}\right)-\frac{p}{J} C_{r}-\frac{F}{J} \omega_{r s} \\
y_{s}= & \omega_{r s}
\end{aligned}
$$

with $\omega_{r s}(0)=0$

Fast model

$$
\begin{aligned}
\frac{\mathrm{d} i_{d f}}{\mathrm{~d} \tau} & =-\varepsilon \frac{R_{s}}{L_{d}} i_{d f}+\varepsilon \frac{L_{q}}{L_{d}} \omega_{r 0} i_{q f}+\frac{\varepsilon}{L_{d}} v_{d f} \\
\frac{\mathrm{d} i_{q f}}{\mathrm{~d} \tau} & =-\varepsilon \frac{R_{s}}{L_{q}} i_{q f}-\varepsilon \frac{L_{d}}{L_{q}} \omega_{r 0} i_{d f}+\frac{\varepsilon}{L_{q}} v_{q f}-\varepsilon \frac{\varphi_{f}}{L_{q}} \omega_{r 0} \\
y_{f} & =i_{d f}
\end{aligned}
$$

with $i_{d s f}(0)=0$, and $i_{q s f}(0)=0$.

$$
u=\left[\begin{array}{l}
v_{d s} \\
v_{q s}
\end{array}\right]^{t}=\left[\begin{array}{l}
v_{d s s}+v_{d s f} \\
v_{q s s}+v_{q s f}
\end{array}\right]^{t} .
$$

A judicious choice of the controls such as

$$
\begin{aligned}
& v_{d s}=v_{d s f} \text { with } v_{d s s}=0, \\
& v_{q s}=v_{q s s} \text { with } v_{d s f}=0
\end{aligned}
$$

deals to the two decoupled models according to

Slow model:

$$
\begin{gathered}
\frac{\mathrm{d} \omega_{r s}}{\mathrm{~d} t}=\frac{p^{2} \varphi_{f}}{J\left(\frac{R_{s}^{2}}{L_{q} L_{d}}+\omega_{r s}^{2}\right)}\left(\frac{R_{s}}{L_{d} L_{q}} v_{q s}-\frac{R_{s} \varphi_{f}}{L_{d} L_{q}} \omega_{r s}\right) \\
-\frac{p}{J} C_{r}-\frac{F}{J} \omega_{r s}
\end{gathered}
$$

$$
y_{s}=\omega_{r s}, \quad \text { with } \quad \omega_{r s}=0 .
$$

Fast model:

$$
\begin{aligned}
\frac{\mathrm{d} i_{d f}}{\mathrm{~d} \tau} & =-\varepsilon \frac{R_{s}}{L_{d}} i_{d f}+\frac{\varepsilon}{L_{d}} v_{d f}, \\
\frac{\mathrm{d} i_{q f}}{\mathrm{~d} \tau} & =-\varepsilon \frac{R_{s}}{L_{q}} i_{q f}, \\
y_{f} & =i_{d f}, \quad \text { with } \quad i_{d f}(0)=0 .
\end{aligned}
$$

\section{THE LOAD TORQUE ESTIMATE}

The load torque is hardly measurable what obliges us to use its estimate in the $I_{q c}$ control expression. The method suggested by le Pioufle permits to estimate in real time the load couple [2].

Figure 3 illustrates the estimator principle.

The error between measured speed and estimated speed is presented as an input of a regulator PI whose output is

$$
\tilde{C}_{r}=\frac{1+\frac{k_{1}}{k_{2}} s}{1+\frac{1+k_{1}}{k_{2}} s+\frac{1}{k_{2}} s^{2}} C_{r},
$$

$k_{1}$ and $k_{2}$ are determined by the poles placement method.

The estimated torque follows with a good precision the load torque variations static mode while in dynamic mode it presents a light shift due to the estimator reaction. 


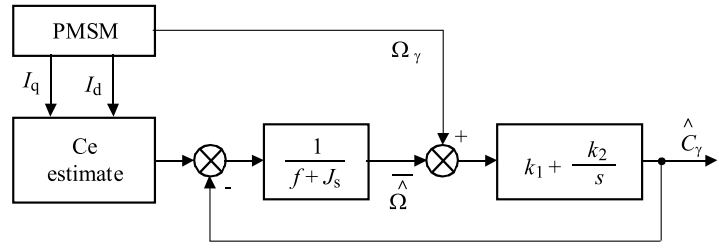

Fig. 1. Load torque estimator

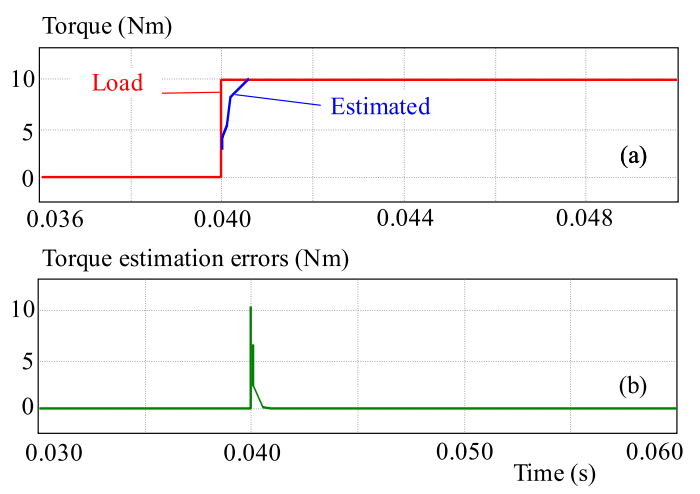

Fig. 2. The estimator response characteristic

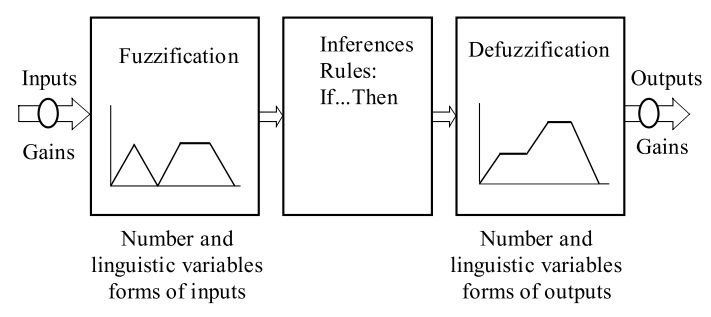

Fig. 3. Internal FLC structure
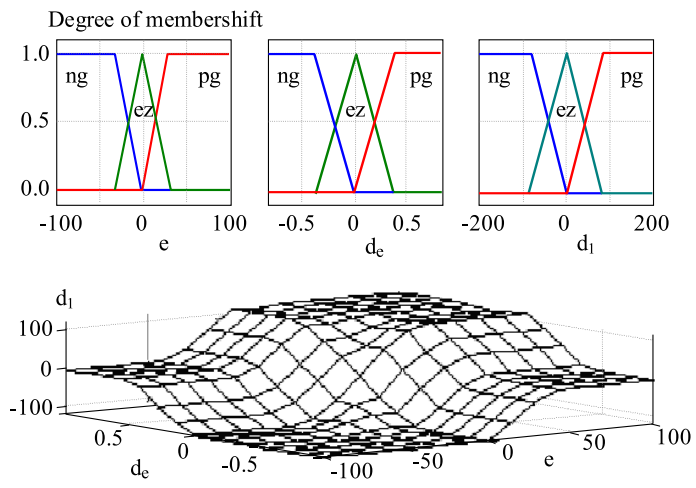

Fig. 4. Membership function and surface control

\section{THE FUZZY LOGIC CONTROL (FLC)}

\subsection{Fundamentals of FLC}

The schematic diagram of a closed loop FLC for PMSM drive is shown in Fig. 6 .

The FLC has three functional blocks for calculation and knowledge base which contains two databases. The functional bloks in FLC are
- Fuzzification is defined as the mapping from a real valued point to fuzzy set. In most fuzzy decision systems, non fuzzy input data is mapped to fuzzy sets by treating them as triangular membership functions, gaussian membership functions, ...

- Inference mechanism. Fuzzy inference is used to combine the fuzzy IF-THEN in the fuzzy rule base, and to convert input information into output membership functions. An inference mechanism emulates the experts decision making in interpreting and applying knowledge about how to perform good control. The rule may use the experts experience and control engineering knowledge. There are three types fuzzy rule based models for function approximation. Mandani model, Takagi-sugeno model,and Koskos addition model.

- Defuzzification. There are many methods which can be used for converting the conclusions of the inference mechanism into the actual input for the plant. Center of gravity defuzzification method is often used. Other defuzzification strategies can be found in technique literatures.

\subsection{FLC design}

The inputs to the fuzzy controller are error $(e)$ and variation error $(d e)$. The output of the fuzzy controller is $(d u)$.

The universe of $(e),(d e)$, and $(d u)$ are partitioned into three fuzzy sets. $N$ (negative), $Z$ (zero), $P$ (positive). Each fuzzy set is represented by triangular membership function or trapezoidal membership function (Fig. 4).

The rule base of the FLC contains nine rules based on the IF-THEN structure which are tabulated in Table 1.

The crisp output of the FLC is obtained by using Max-Min inference algorithm and the center of gravity defuzzification approach. It is known that the sensitivity and performance of FLC system are significantly affected by the weighting factors $C_{1}, C_{2}$ and $C_{3}$. However, we lack a general methodology for choosing these factors. In this paper, a trial and error approach is used to determine and adjust these weighting factors [4-6].

\section{THREE LEVELS INVERTER MODELLING}

The NPC three levels inverter of tension consists of twelve pairs of transistors - diodes that generate levels of amplitude tension $-U, 0, U$. It is generally controlled by the PWM. The simple tension of each phase is entirely defined by the state of the four transistors (Switches) constituting each arm. The median diodes of each arm permits to have the zero level of the inverter output voltage. Only three sequences of operation are retained and done in work. Each arm of the inverter is modelled by a perfect switch with three positions $(-1,0,1)$ (Fig. 5). The operation of the converter is based on the PWM strategy with two carriers. The intersections of these last with the modulating signals determine the instants and the durations 


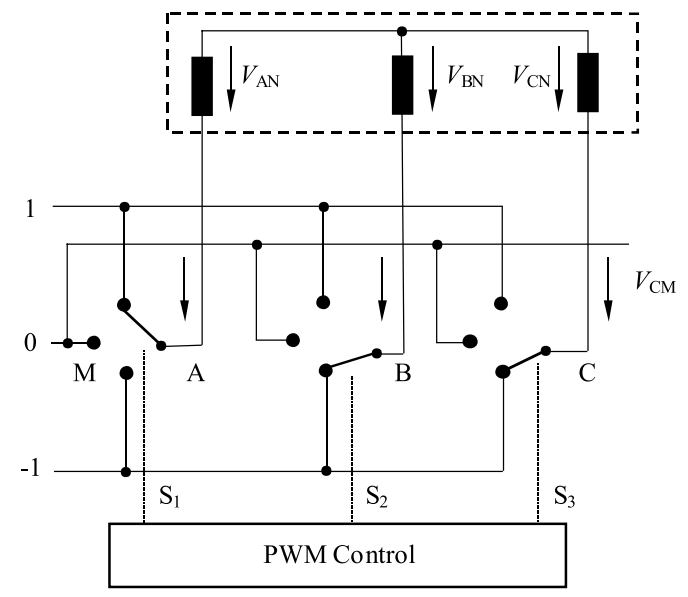

Fig. 5. Functional diagram of the Multilevel Inverter

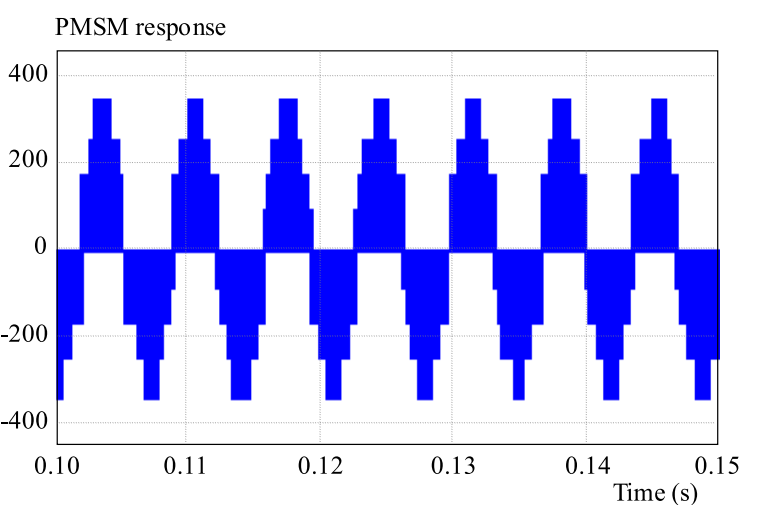

Fig. 7. PMSM responses to a level speed of 100rd/s followed by an inversion speed $-100 \mathrm{rd} / \mathrm{s}$ at $.8 \mathrm{~s}$

of closing or opening of the switches of each arm [10]. The three-phase simple power provided by the inverter is determined by the following relation.

Table 1.

\begin{tabular}{ccccc}
\hline \multicolumn{1}{l}{$d u$} & \multicolumn{3}{c}{$e$} \\
& & $N$ & $Z$ & $P$ \\
\cline { 3 - 5 }$\Delta e$ & $N$ & $N$ & $N$ & $Z$ \\
& $Z$ & $N$ & $Z$ & $P$ \\
& $P$ & $Z$ & $P$ & $Z$ \\
\hline & \\
& \\
&
\end{tabular}

with

$\left(\begin{array}{lll}V_{a n} & V_{b n} & V_{c n}\end{array}\right)^{t}=[V],\left(\begin{array}{lll}S_{1} & S_{2} & S_{3}\end{array}\right)^{t}=[S]$,

$[C]=\left(\begin{array}{c}2 ;-1 ;-1 \\ -1 ; 2 ;-1 \\ -1 ;-1 ;-1 ; 2\end{array}\right)$,

$S_{i}=T_{i 1} T_{i 2}-T_{i 3} T_{i 4}, ;(i=1,2,3)$,

$S_{i}$ : Logic signals (PWM),

$F_{i}$ : Switching functions.

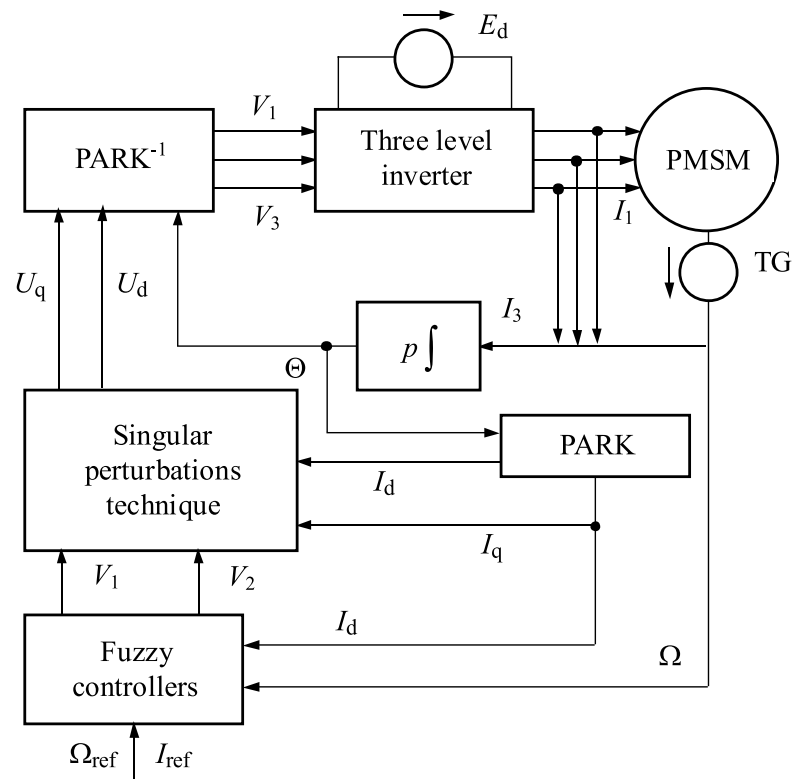

Fig. 6. General Diagram of the fuzzy control with decoupling based on the singular perturbations technique of the PMSM

\section{SIMULATION}

The decoupling based on the singular perturbations technique control of the PMSM supplied with a three levels inverter of tension (PWM) and with the fuzzy control (Fig. 6), is tested by digital simulation.

Figure 7 represents the performances of the control device proposed for a speed level of 100rd/s followed by an inversion of speed rotation $-100 \mathrm{rd} / \mathrm{s}$ at $.8 \mathrm{~s}$. The control performances are very satisfactory. The dynamics of continuation is not affected during the inversion of the speed. The decoupling is ensured when inversing the speed. One notices, for speed, a starting without an overshoot and static error and a fast perturbation rejection. The $I_{d}$ current is maintained null and independent of the speed inversion (torque). The $I_{q}$ current is proportional to the torque. The fluctuations recorded on the currents are due to the inverter control.

\section{CONCLUSION}

We presented in this paper the fuzzy control performances for a PMSM decoupled by the singular perturbations technique and associated to a three levels inverter (PWM). The results obtained show the applicability of this control technique in the field of the electric drives. The objective of continuation is very good. The speed time response and the perturbation rejection are very good with no overshoot.

Decoupling is maintained even with the inversion of the speed. The singular perturbation technique permits to bring the behaviour of the closed loop system of a nonlinear system to a decoupled linear system. This control strategy provided a stable system with satisfactory performances with a good decoupling. 

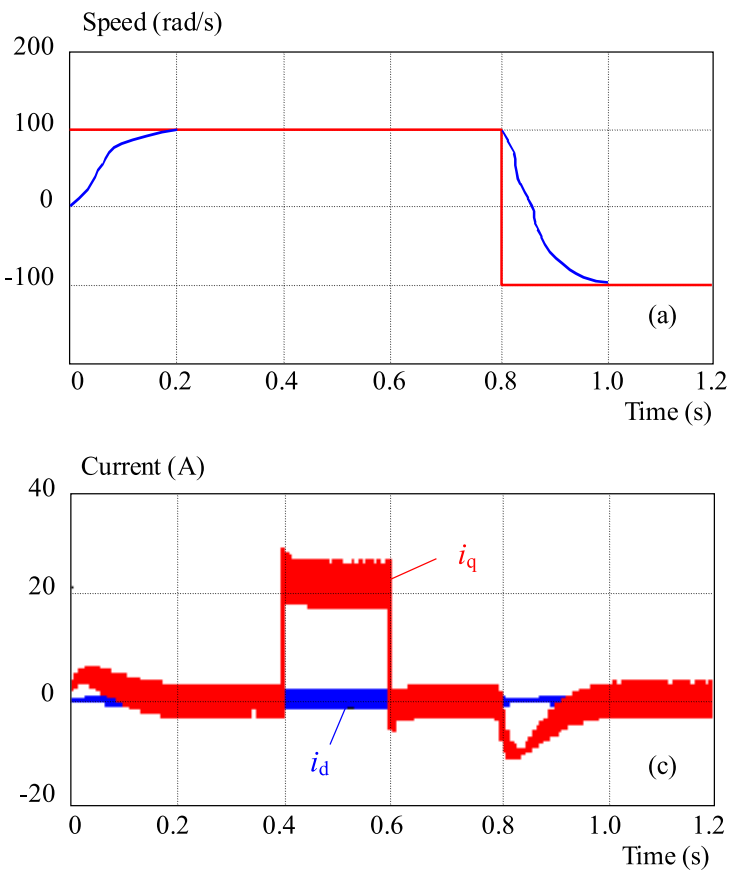
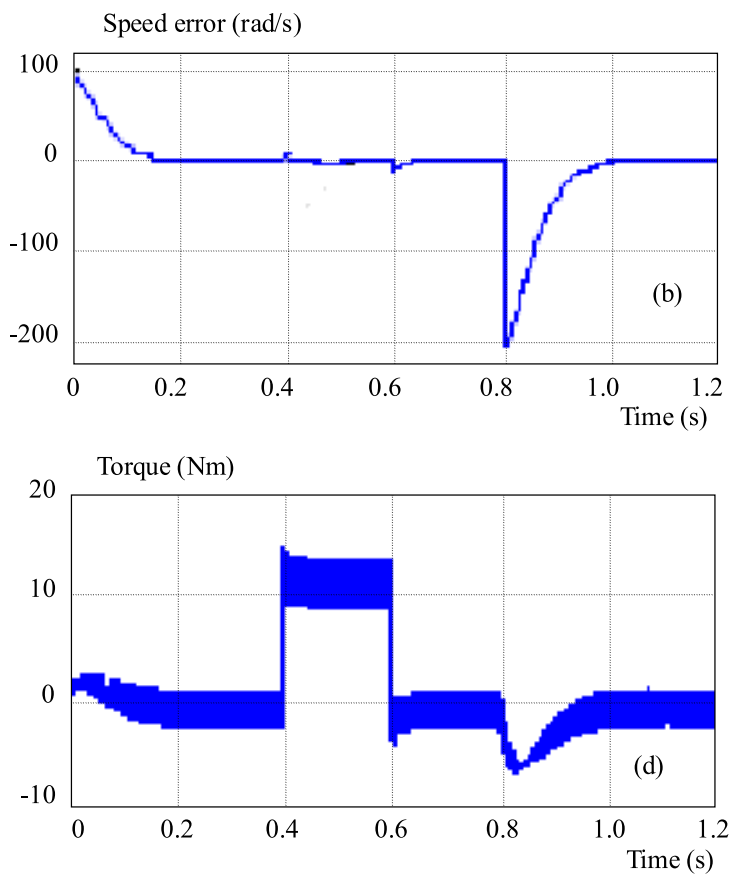

Fig. 8. PMSM responses to a level speed of $100 \mathrm{rd} / \mathrm{s}$ followed by an inversion speed $-100 \mathrm{rd} / \mathrm{s}$ at $0.8 \mathrm{~s}$

\section{REFERENCES}

[1] LEONHARD, W. : Control of Electrical Drives, Springer Verlag, Berlin, 1985.

[2] KAPOLINO, G. A.: Vector Numerical Control Of the Current AC Machines, RGE NO5 (May 1992), 148-160.

[3] MAKATO IWASAKI-NOBUYUKI MATSUI : Robust Speed Control of IM with Torque Feed Forward Control, IEEE Trans. Ind. Elect. 40 No. 6 (Dec 1993), 553-560.

[4] MORERE, Y.: Neuro-Fuzzy Networks, May 17.

[5] MASSOUM, A.: Fuzzy Control of the Asynchrone Machine Fed by a Three Level Inverter with the Rotoric Time Constant Correction, Canada, 2003.

[6] KOIVO, H. : Fuzzy Exercises, 2000.

[7] SONGT, T.-RAHMANT, M. F.-LIMT, K. W.-RAHMANTI, M. A.: A Singular Perturbation Approach to Sensorless Control of a Permanent Magnet Synchronous Motor Drive, IEEE Transactions on Energy Conversion 14 No. 4 (Dec 1999).

[8] TSANG, K. M.-WANG, J.: Design of Second Order Sliding Mode Controller for Synchronous Generator based on Singular Perturbation Method, IEEE, 2002.

[9] SOTO-COTA, A.-LOUKIANOV, A.-CAIIED, J. M.-FRIDMAN, L. M. : Variable Structure Control of Synchronous Generator: Singularly Perturbed Analysis, Proceedings of the $42^{\text {nd }}$ IEEE Conference on Decision and Control, Maui, Hawaii USA, Dec 2003.

[10] BUMP, B. K.: Power Electronics and AC Drives, Printice Hall, New York, 1986.

Received 21 December 2010

Ahmed Massoum was born in 1959 in M'sirda Fouaga, Tlemcen, Algeria. He received his BS degree in electrical engineering from the Electrical Engineering Institute (INELEC) of Boumerdes 1985 and the MS degree from the Electrical Engineering Institute of Sidi Bel-Abbes University in 2004 where he is currently full professor of electrical engineering. He is a member of Intelligent Control Electrical Power System Laboratory (ICEPS). His current research interest includes power electronics and drives.

Patrice Wira received the MSc degree and the $\mathrm{PhD}$ degree in Electrical Engineering from the University of Haute Alsace, Mulhouse, France, in 1997 and 2002, respectively. He received the Accreditation to Supervise Research (the French Habilitation Diriger des Recherches) in computer sciences from the University of Haute Alsace in 2009. He was an associate professor with the MIPS Laboratory (Laboratoire Modlisation, Intelligence, Processus, Systéme) at the University of Haute Alsace. Since 2011, he is a full professor. He is author or coauthor of more than 20 technical papers covering his research interests from artificial neural networks applied to the modelling and simulation of complex automation systems, to neuro-control approaches and other adaptive control systems.

Abdelkader Meroufel was born in Sidi Bel-Abbes (Algeria) 1954. He received his BS degree in electrical engineering from USTOran in 1979. He is a member of Intelligent Control Electrical Power System Laboratory (ICEPS). His current research interest includes power electronics and drives.

Mohammed Karim Fellah was born in Oran, Algeria, in 1963. He received the Eng degree in Electrical Engineering from University of Sciences and Technology, Oran, Algeria, in 1986, and the PhD degree from National Polytechnic Institute of Lorraine (Nancy, France) in 1991. Since 1992, he is full professor at the University of Sidi-bel-Abbes (Algeria) and director of the Intelligent Control and Electrical Power Systems Laboratory at this University. His current research interest includes power electronics, HVDC links, and drives. 